\title{
Sound Energy Harvesting Methods
}

\author{
Dande Chandra Sekhar Charan, Bhooshan Rajpathak
}

\begin{abstract}
This paper presents the various strategies and aspects of sound energy harvesting. We have discussed sound energy harvesting related to wireless sensor networks(WSNS) and regarding ultra low power requirements.
\end{abstract}

Keywords: Piezo electric material, Sound Energy harvesting, Wireless sensor networks

\section{INTRODUCTION}

In the age of information, with the demand of energy increasing day by day, energy harvesting has gained the prime spot in the field of research. With the digital revolution and emerging fields like e-mobility, 'Battery' has become the key to solve most of the problems in energy management. Due to limitations in charging of the battery by conventional sources, the alternative to conventional battery charging is most sought after. Many authors have explored this problem [12]. In some situations, if the battery is dead it becomes very difficult to supply power to the remote locations and also requires high maintenance under such situations. To counter these situations, it is essential to think of energy harvesting through various sources present around us.

The noise pollution is one of the major pollution and it is profitable if we are able to convert such pollution into energy, a source for battery charging. Sound to electricity conversion is thus an idea worth exploring. It is like tackling two problems, environmental pollution and energy harvesting at the same time [11].

We have reviewed various concepts related sound energy harvesting and generated a Simulink model through which various results are deduced and presented in the paper.

\section{SOUND ENERGY HARVESTING FOR WSNS}

Now a day we are entering into the era of Internet of things. Internet of things can be explained as an interconnection between the devices via internet which enables us to send and receive the data. It basically helps in making the day today life lot simpler.

The main component of Internet of things is the wireless sensor networks which are supplied with power either through direct contact or indirect contact. Generally, they are powered through batteries which have a disadvantage of limited lifespan. In this case sound energy harvesting can be a handy, which can be act as a semipermeable alternate power supply.

The sound energy(noise) produced in the environment of the sensor can be utilized for the powering up the sensor. For example, if the sensor is located in an environment of a machine for some purpose, we can utilize the noise produced by the machine to power up the sensor.

\section{A. Basic Sound Energy Harvester}

There are various methods that can be used for the conversion of sound to electricity. One of which is by utilizing the piezo electric materials.

As the sound can be treated as a pressure wave travelling through a medium, so when they are made to concentrate on the sound energy harvester, then the voltage is produced which can be trapped to produce electricity.

Sound pressure level (SPL), which is equivalent to to sound expressed in terms of pressure, is defined as:

$\mathrm{SPL}=20 \log \mathrm{p} / \mathrm{po}($ expressed in $\mathrm{dB})[8]$.

Basic sound energy harvester consists of Resonator. Membrane and Piezo electric material.

Resonator is used to amplify the sound signal, the process of amplification is carried out because the sound wave has very low energy density. Three types of resonators can be used in sound energy harvesting.

- Helmholtz resonator

- Quarter wavelength resonator

- Half wavelength resonator
Revised Manuscript Received on July 22, 2019.

Dande Chandra Sekhar Charan, Electrical Department, VNIT, Nagpur, India.

Bhooshan Rajpathak*, Electrical Department, VNIT, Nagpur, India.
Published By:

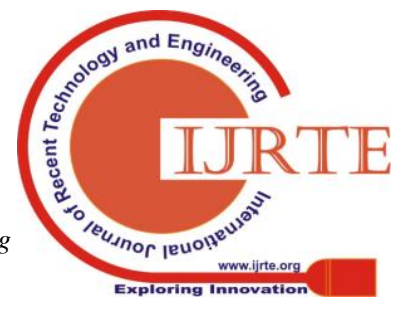




\section{Sound Energy Harvesting Methods}

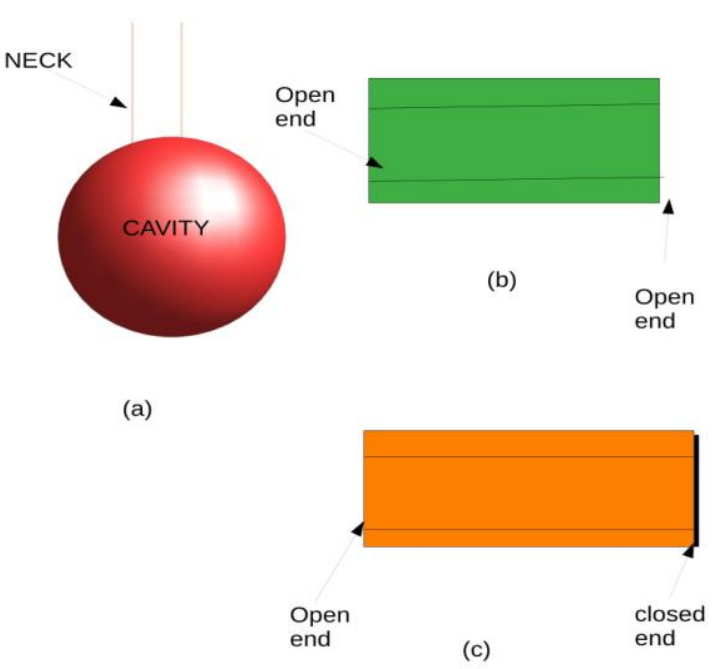

Helmholtz resonator has a large volume connected through a narrow neck. Quarter wavelength resonator is open at one end and closed at other end whereas half wavelength resonator is open at both the ends.

It was observed that quarter wavelength resonator is said to have twice the large max absorption coefficient than half wavelength resonator at normal frequency range of $560 \mathrm{~Hz}$ and same design parameters [6]. For same design parameters like volume, quarter wavelength resonator has a larger maximum sound absorption coefficient compared to Helmholtz. So Quarter wavelength resonator can be treated as the best resonator among the other types to collect the sound energy at a particular frequency.

Now the amplified sound is transferred membrane i.e., the membrane which is attached to one end oscillates at the same frequency. The other end of membrane is attached to the piezoelectric material, so due to the vibrations of the membrane, there is force acting upon piezoelectric material and a voltage is generated.

\section{B. Electromagnetic Sound Energy Harvester}

This sound energy harvester eliminates the use of piezo electric material. It works based on the Faraday's law of electromagnetic induction. Here if the vibrations of the membrane (which oscillates at the same frequency of the resonator) are transferred on the coil present in the between two magnets (electromagnets or permanent magnets), due to motion of the coil an emf is induced which can be used to generate the electricity

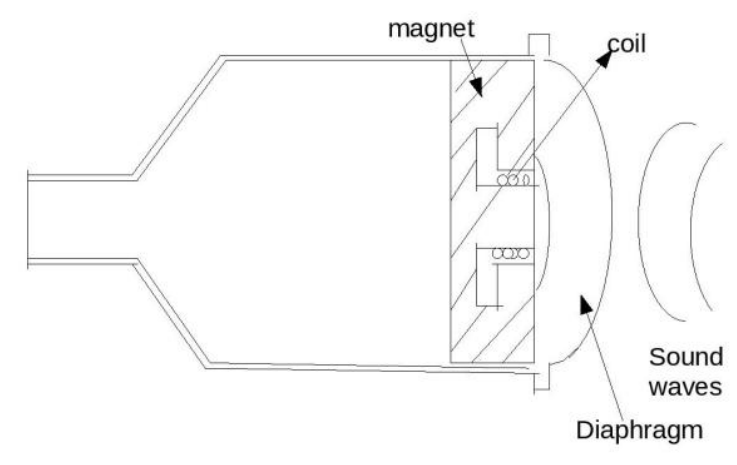

\section{Acoustic metamaterial Sound Energy Harvesting}

There is another type of sound energy harvesters called acoustic meta materials based sound energy harvesting. These are designed in such a way that they can control and manipulate sound waves at a particular frequency which are generally not observed in nature. The mechanism involved by which they control the sound waves is that they create an artificial periodic geometry by using materials like metal and plastic materials. The main advantage of these materials is that it makes it possible to decrease the dimensions of the device for low frequency harvesting devices, this is because in resonance only depends on the geometry and the material properties but not on the dimension of the device.

There are two (major) types of acoustic meta materials:

- Phononic Crystal

- Sonic Crystal

Phononic crystal: It is a artificially made periodic structure which is made from sonic scatters present in host material.

Sonic crystal: It was proposed by Liu et al. It is made of periodic arrangement of sonic scatters having different mechanical properties.

It is advisable to prefer the resonator (Quarter wavelength resonator) instead of acoustic meta materials as the output obtained was low in case of acoustic meta material compared to resonator. The main advantage of acoustic meta material is that device does not depend on dimension.

\section{Use of Capacitor like plates}

The main components of this device are metal diaphragm and a metal electrode (which is kept extremely close to diaphragm). The electrode and the diaphragm are mutually insulated and they can be treated as a plates of a capacitor. For compensating the high sound pressure, a small hole is punched on the wall.

Whenever the device is exposed to the sound, the path present between diaphragm and electrode is affected by force (directly proportional to pressure of sound and area of diaphragm). The movement of diaphragm causes change in the capacitance, which in turn causes change in voltage. This voltage can be trapped to store energy or can be used instantaneously. [8] 


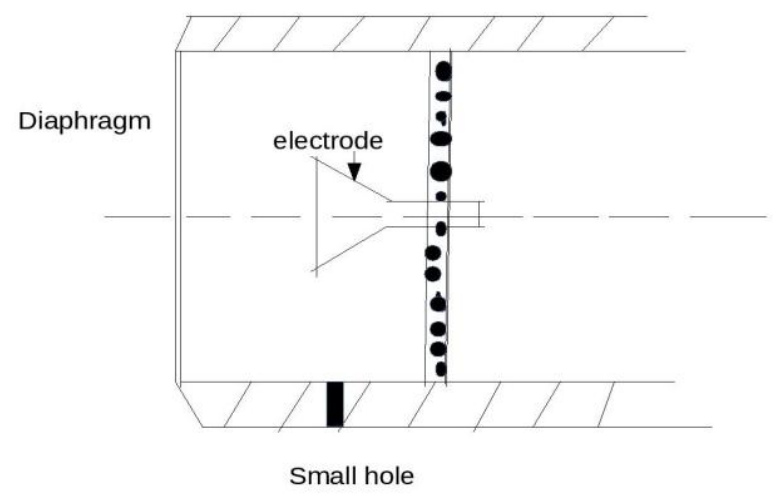

\section{PIEZO-ELECTRIC MATERIALS}

Piezo electric materials are those which generate potential

\begin{tabular}{|c|c|}
\hline $\begin{array}{l}\text { Numerical difference } \\
\text { between noises }\end{array}$ & $\begin{array}{l}\text { Amount of dB added } \\
\text { to higher noise level }\end{array}$ \\
\hline $6.1-10$ & 0.5 \\
\hline $4.1-6$ & 1.0 \\
\hline $2.4-4$ & 1.5 \\
\hline $1-2.4$ & 2.0 \\
\hline $0.1-0.9$ & 0.5 \\
\hline
\end{tabular}

when a mechanical force is applied. So when the sound waves are focused on the piezoelectric materials, due their pressure, voltage is produced.

Examples of Piezo-electric materials are Quartz, artificial Lithium Niobate crystal, Lead Zirconate Titanate(PZT). It is found that artificial Lithium Niobate crystal converts sound to electricity only under the conditions of high temperature and frequency. Whereas PZT has no such restriction. Comparable to Quartz, PZT was proven to be advantageous in generation of electricity. As the PZT is extensively used as piezoelectric material, there was extensive research to find $\mathrm{Pb}$ free material. Fortunately, a ceramic comparable to PZT in piezoelectric characteristics has been discovered. This ceramic is BT (Barium Titanate). It was reported that $\mathrm{Ba}(\mathrm{Zr}, \mathrm{Ti}) \mathrm{O} 3$-(Ba,Ca)TiO3 (BZT-BCT) has a large piezoelectric response with $\mathrm{d} 33$ coefficient comparable to PZT ceramic. This means, having high sensitivity and permittivity $[1,2]$.

The primarily used piezoelectric materials in the research study of sound energy harvesting are 20-A4-503YB and EH220-A4-503YB. They are efficient enough for the use in sound energy harvesting. This is due to their large size and larger surface area, which enables them to observe more sound waves.

As the output of piezoelectric material will still be noisy it can be stored in super capacitors. This super-capacitor will be connected in parallel to piezoelectric material, it acts as both for filtering purpose and to store the produced electricity. It is also called as Electrical double layer capacitor(ELDC).

\section{FOR INSTANTANEOUS USAGE}

If at all the output needed to be used for instantaneous purpose, then we need to ensure that the supply of electricity is constant even when there is not enough sound. So for this purpose, a battery and main power supply is also used. Main power supply is used only for emergency when no enough sound is present and battery is dead. Main power supply can also be used to charge the battery when it gets discharged. Voltage regulator is also present in order to provide constant voltage supply.

In practice, $\mathrm{DC} / \mathrm{DC}$ converters are used to contain large and expensive electromagnetic components to reduce wiring and conduction losses. A new approach to maximize output power of piezoelectric energy harvester is by resistance matching which is done by the use of step down piezoelectric transformer (PT) [13]. Here the large and expensive electromagnet are replaced by ceramic component which does not use any electromagnetic interference. This technique reduces the circuit size and this transformer can be manufactured on the cantilever beam. In order to operate PT at its resonant frequency, half bridge driving circuit can be used. It should be noted that it has no inductive component $[3,9]$.

\section{SOUND HARVESTING FOR HIGHER VOLTAGE REQUIREMENTS}

Recently an inventor of Boeing company filed a patent regarding harnessing the noise (a form of sound energy) from the aircraft into usable electrical energy. This is achieved by the use of acoustic electricity generator. As the noise produced by aircraft is large there no need of any voltage amplifiers. It was reported that the electricity produced in such way was sufficient enough to light the airport.

The same idea can be incorporated for the producing the electricity at the highways and also the device can be attached to the vehicle itself, now the electricity is produced not only due to the noise produced but also due to the air drag that opposes the car during the motion. This electricity produced can be utilized for the various purposes like charging the mobile phones etc. (Please refer to the graph added on last page depicting the performance of various piezoelectric materials.)

The noise level observed near the machines like textile loom etc at the distance of 1 meter is $103 \mathrm{~dB}$ and at the newspaper press is observed to be $95 \mathrm{~dB}$.

The sound energy increases by more than $6 \mathrm{~dB}$ by decrease in 


\section{Sound Energy Harvesting Methods}

half of the distance. So, the range of sound level that can be observed at the closest proximity is taken from a range of 100 to $200 \mathrm{~dB}$. The result of the voltage that got produced can be observed in the graph.

It is also observed that the noise level produced by the car travelling at $60 \mathrm{kmph}$ at a distance of 20 is $65 \mathrm{~dB}$ and a truck moving at $50 \mathrm{kmph}$ at a distance of $20 \mathrm{~m}$ is $85 \mathrm{~dB}$. This data proves that incorporation of Sound energy harvesters is profitable, as the produced electricity can be utilized for the street lights.

It is also observed that when there two noises present at the same time the difference in the noises, increase the amount of $\mathrm{dB}$ to noise with higher $\mathrm{dB}$.

Now for actually using the harvester for the storage of energy, we can use a power amplifier.

\section{RESULT AND DISCUSSION}

Hence from the results obtained, it can be observed output voltage obtained is good enough and in the extreme cases for high power requirement power amplifier can be used. Hence, the sound energy harvester can be used to power up the sensors from the energy that is actually getting wasted and also for higher voltage requirements as specified above.

The same methods can be used for:

- Sound energy harvesting in substations is suggested by [5]. Here the noise is mainly produced by the working transformer and the reactor. It is observed that average noise level of a substation is around 80 to $100 \mathrm{~dB}$. It is also found that frequency range in which noise is concentrated is around 50 to $500 \mathrm{~Hz}$, which is a low frequency.

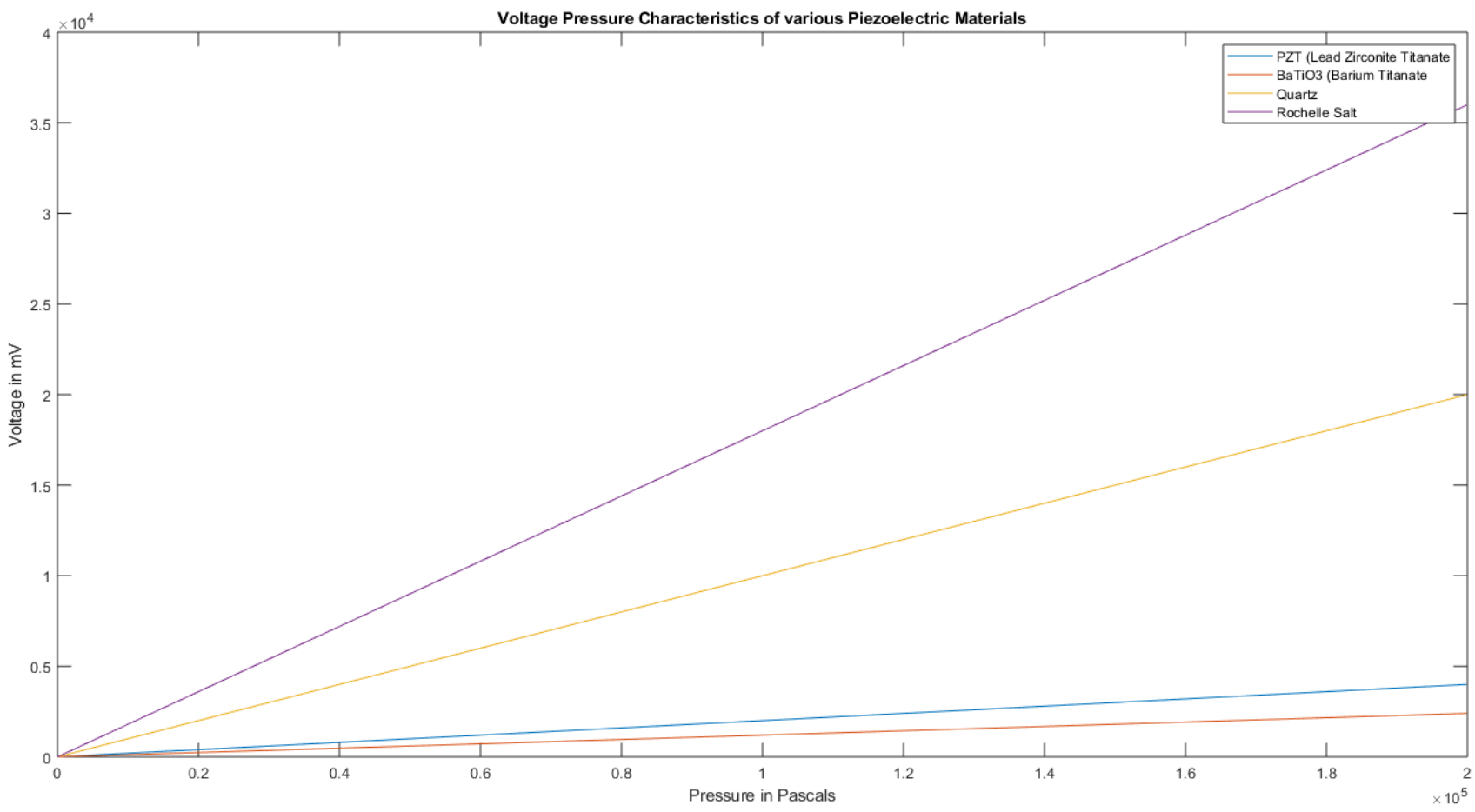

As we know low frequency waves have strong diffraction and transmission capacity, so they can easily pass through any obstacle and these will be having a large influence on the workers. This sound level also increases with increase in the voltage grade on which the substation is operating. So, by using this noise we can produce electricity and this will also reduce the influence of noise on the workers. As the substations operate throughout 24 hours, source of the noise is stable and electricity is produced at a constant supply throughout the day.
- Even large amount of sound is produced from the train wheels, which can be converted into useful energy and practically it was proven to have good results [4].

- We can use the same method to convert sound to electricity in the stadium. In stadium enough noise is produced that can be converted into electricity.

\section{CONCLUSION}

Sound energy harvesting is essential as well as economical 
alternative to exploring renewable energy sources. Both, noise pollution and energy harvesting are the positives of this process. Sound energy harvesting through piezoelectric is more interesting as we can have energy storage and instantaneous energy use applications. For large energy requirement, aircraft or railway can provide the solution.

\section{REFERENCES}

1. A, M.O., S, R., K, R., D, R.: Generation of electricity from industrial noise. International Research Journal of Engineering and Technology (IRJET) pp. 818-820 (2016)

2. Attia1, M.F.S., Abdalateef, A.I.M.: Evaluation of electric energy generation from sound energy using piezoelectric actuator. International Journal of Science and Research (IJSR) pp. 218-225 (2014)

3. Bhatnagar, S.R.: Converting sound energy to electric energy0. International Journal of Emerging Technology and Advanced Engineering (2012)

4. Billah, M.M., Ullah, M.R., Alam, M.A., Nondy, R.K., Alam, F., Mishnu, A.P.: Generation of electrical energy using piezoelectric material from train wheels: Bangladesh perspective. In: The 9th International Forum on Strategic Technology (IFOST). Bangladesh (oct 2018)

5. Chen, C., YangGao, SongtaoLiu: Study on noise prediction model and control schemes for substation. The Scientific World Journal pp. 1-7 (Feb 2014)

6. Choi, J., Jung, I., Kang, C.Y.: A brief review of sound energy harvesting, nano energy. scientific world (Nov 2018)

7. Ebrahimi, N.D., Wang, Y., Ju, Y.: Mechanism of power dissipation in piezo electric fans and their correlation with convective heat transfer performance. Elsevier pp. 242-252 (2018)

8. Ge, Q.: Prospect of electric generation using sound. In: AIP Conference Proceedings 1839, 020050 (2017). pp. 1-5. American Institute of Physics (2017)

9. Joshi, H., Zah, M.M., Jeevansingh, B.G., Mehboob, P.S.: Alternative form of power generation. International Journal for Scientific Research and Development pp. 101- 103 (2018)

10. Kulkarni, P., Tandsi, A., Bopanna, D., Kamath, N.: Analysis of conversion of noise to electrical energy in a stadium. International Journal of Industrial Electronics and Electrical Engineering pp. 1-4 (2017)

11. Pujari, M., Chandra, S., Dibya, K., Chandra, S., Patel, B., Neha, K. Electricity generation by noise. International Journal of Scientific Research in Computer Science, Engineering and Information Techn pp. 369-371 (2017)

12. Rajule, N., Gowhar, S., Pawar, S., Raut, A.: Talk and charge. International Journal of Electrical and Electronics Engineers pp. 419-426 (2015)

13. Vivek, M.: Sound energy generates electricity: An exploratory study. International Journal of Electronics, Electrical and Computational System pp. 56-59 (2017)

\section{AUTHORS PROFILE}

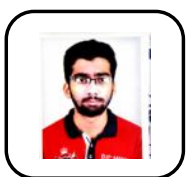

Dande Chandrasekhar Charan is an undergraduate student in the department of Electrical engineering at VNIT Nagpur. He has published one conference paper at international level. His research interests are power systems, control system, transducers.

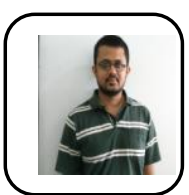

Dr. Bhooshan Rajpathak is Assistant professor in the department of Electrical engineering at VNIT Nagpur. He has published 4 international journal papers and 7 international conference papers. His research interests are control theory, non-linear systems, transducers. 